Pacific Journal of Mathematic

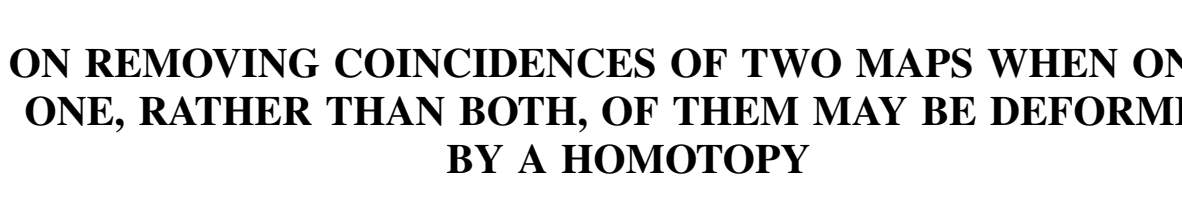




\title{
ON REMOVING COINCIDENCES OF TWO MAPS WHEN ONLY ONE, RATHER THAN BOTH, OF THEM MAY BE DEFORMED BY A HOMOTOPY
}

\author{
RoBin B. S. Brooks
}

It is known that if $f, g: X \rightarrow Y$ are maps of a topological space $X$ into a topological manifold $Y$, and that $f$ and $g$ can be deformed by homotopies to maps $f^{\prime}$ and $g^{\prime}$ which are coincidence-free, then $f$ may be deformed by a homotopy to a map $f^{\prime \prime}$ such that $f^{\prime \prime}$ and $g$ are coincidence-free. This result is generalized as follows:

If $f, g: X \rightarrow Y$ are maps of a topological space $X$ into a topological manifold $Y$ and $f^{\prime}$ and $g^{\prime}$ are homotopic to $f$ and $g$ respectively, then for any homotopy $\left\{g_{t}\right\}$ from $g$ to $g^{\prime}$, there is a homotopy $\left\{f_{t}\right\}$ from $f^{\prime}$ such that the set of coincidences of $f_{t}$ and $g_{1-t}$ is the same for all $t \in[0,1]$.

Some applications of this result to fixed point theory and root theory are indicated.

A coincidence of two maps $f, g: X \rightarrow Y$ of topological spaces is a point $x \in X$ such that $f(x)=g(x)$. Denote the set of all such points by $\Gamma(f, g)$. It may be possible to deform both $f$ and $g$ by homotopies to maps $f^{\prime}$ and $g^{\prime}$ such that $\Gamma\left(f^{\prime}, g^{\prime}\right)$ is empty, but still impossible to deform just $f$ to a map $f^{\prime \prime}$ such that $\Gamma\left(f^{\prime \prime}, g\right)$ is empty. This is the case, for example, when $X=Y$ is the unit interval, $g$ is the identity, and $f$ is arbitrary. However, Fuller [4] has shown that when $Y$ is a manifold, the ability to free $f$ and $g$ of coincidences by deforming both $f$ and $g$ is equivalent to being able to free $f$ and $g$ by deforming just $f$. The purpose of this paper is to generalize Fuller's result by showing that (in that case where $Y$ is a manifold) any change in the coincidence set $\Gamma(f, g)$ that may be effected by deforming both $f$ and $g$ can also be effected by deforming just $f$. Precisely, we establish the following theorem.

THEOREM 1. Suppose $f, g: X \rightarrow Y$ maps of a topological space $X$ into a topological manifold $Y$, and let $f^{\prime}$ and $g^{\prime}$ be homotopic to $f$ and $g$ respectively. Then there is a map $f^{\prime \prime}$ homotopic to $f^{\prime}$, and therefore to $f$, such that $\Gamma\left(f^{\prime \prime}, g\right)=\Gamma\left(f^{\prime}, g^{\prime}\right)$. In fact, given any homotopy $\left\{g_{t} \mid t \in I\right\}$ from $g$ to $g^{\prime}$, there is a homotopy $\left\{f_{t} \mid t \in I\right\}$ beginning at $f^{\prime}$ such that $\Gamma\left(f_{t}, g_{1-t}\right)=\Gamma\left(f^{\prime}, g^{\prime}\right)$ for all $t \in I$.

Here $I$ denotes the closed unit interval. 
This theorem (with a weaker hypothesis on $Y$ ) is proved in $\S 2$. The main tool used in its proof is the theory of numerable bundle pairs. This theory is reviewed in the first section of the paper. The last section of the paper is devoted to tracing out some of the consequences of the theorem. In particular, we show that when $Y$ is a manifold the local fixed point theory as well as the global theory can be regarded as a special case of the local coincidence theory, and similarly for the local study of roots (study of equations of the form $f(x)=a$, where $a$ is a given point in $Y$ ).

1. Bundle theory. The following treatment is largely an adaptation to the relative case of terminology and results that may be found in [5].

A bundle pair $\left(p: E \rightarrow B, p_{0}: E_{0} \rightarrow B\right)$ over a space $B$ is a pair of maps such that $E_{0} \subset E$ and $p_{0}=p \mid E_{0}$. Two such pairs $(p: E \rightarrow B$, $\left.p_{0}: E_{0} \rightarrow B\right)$ and $\left(p^{\prime}: E^{\prime} \rightarrow B, p_{0}^{\prime}: E_{0}^{\prime} \rightarrow B\right)$ are isomorphic over $B$ if there is an homeomorphism $h: E^{\prime} \rightarrow E$ such that $p^{\prime}=p \circ h$, and $h\left(E_{0}^{\prime}\right)=E_{0}$.

The bundle pair $\left(p, p_{0}\right)$ is locally trivial iff there is an open cover $\left\{U_{\alpha} \mid \alpha \in A\right\}$ of $B$ and a family

$$
\left\{\left(h_{\alpha}: p^{-1}\left(U_{\alpha}\right) \rightarrow U_{\alpha} \times F_{\alpha}, h_{0 \alpha}: p_{0}^{-1}\left(U_{\alpha}\right) \rightarrow U_{\alpha} \times F_{0 \alpha}\right) \mid \alpha \in A\right\}
$$

of pairs of homomorphisms such that $h_{0 \alpha}=h_{\alpha} \mid p_{0}^{-1}\left(U_{\alpha}\right)$ and $\pi_{\alpha} \circ h_{\alpha}(e)=p(e)$ for every $\alpha \in A$ and $e \in p^{-1}\left(U_{\alpha}\right)$. Here $\pi_{\alpha}: U_{\alpha} \times F_{\alpha} \rightarrow U_{\alpha}$ is the indicated projection. The cover $\left\{U_{\alpha} \mid \alpha \in A\right\}$ is called a trivializing cover.

An open covering $\left\{U_{\alpha} \mid \alpha \in A\right\}$ of a space $B$ is numerable if it is locally finite and has a partition of unity subordinate to it. A bundle pair is numerable if it has a numerable trivializing cover. Every locally trivial bundle pair over a paracompact space is numerable.

A bundle pair $\left(p^{\prime}: E^{\prime} \rightarrow B^{\prime}, p_{0}^{\prime}: E_{0}^{\prime} \rightarrow B^{\prime}\right)$ is a pullback under the map $f: B^{\prime} \rightarrow B$ of a bundle pair $\left(p: E \rightarrow B, p_{0}: E_{0} \rightarrow B\right)$ if there is a map $f^{*}:\left(E^{\prime}, E_{0}^{\prime}\right) \rightarrow\left(E, E_{0}\right)$ such that $p\left(f^{*}(e)\right)=f\left(p^{\prime}(e)\right)$ for all $e \in E^{\prime}$, and if $\left(p^{\prime \prime}: E^{\prime \prime} \rightarrow B^{\prime}, p_{0}^{\prime \prime} \cdot E_{0}^{\prime \prime} \rightarrow B^{\prime}\right)$ is any other bundle pair over $B^{\prime}$, and $f^{* *}:\left(E^{\prime \prime}, E_{0}^{\prime \prime}\right) \rightarrow\left(E, E_{0}\right)$ a map such that $p\left(f^{* *}(e)\right)=f\left(p^{\prime}(e)\right)$ for all $e \in E^{\prime}$, then there is an unique map $u:\left(E^{\prime \prime}, E_{0}^{\prime \prime}\right) \rightarrow\left(E^{\prime}, E_{\mathrm{c}}\right)$ such that $f^{* *}=$ $f^{*} \circ u$, and $p^{\prime}(u(e))=p^{\prime \prime}(e)$ for all for $e \in E^{\prime \prime}$. Any two pullbacks of $\left(p, p_{0}\right)$ under $f$ are isomorphic over $B^{\prime}$. Given $f: B^{\prime} \rightarrow B$ one may always construct a pullback $\left(p^{\prime}: E^{\prime} \rightarrow B^{\prime}, p_{0}^{\prime}: E_{0}^{\prime} \rightarrow B^{\prime}\right)$ and a map $f^{*}:\left(E^{\prime}, E_{\mathrm{c}}\right) \rightarrow\left(E, E_{0}\right)$ by letting $E^{\prime}=\left\{\left(b^{\prime}, e\right) \in B^{\prime} \times E \mid p(e)=f\left(b^{\prime}\right)\right\}$, $p^{\prime}\left(b^{\prime}, e\right)=b^{\prime}$ and $f^{*}\left(b^{\prime}, e\right)=e$ for all $\left(b^{\prime}, e\right) \in E^{\prime}, E_{0}^{\prime}=f^{*-1}\left(E_{0}\right)$, and $p_{0}^{\prime}=$ $p^{\prime} \mid E_{0}^{\prime}$.

A pullback of a locally trivial (numerable) bundle pair is locally trivial (numerable).

The main result we need from bundle theory is Theorem 2 below. 
The corresponding result for principle $G$ bundles is proved in [5, p. 50]. The same proof carries over to the case of numerable bundles, if one substitutes Exercise 5 on page 22 of [5] for Lemma 4.1 of [5]. The proof for numerable bundle pairs is essentially the same as the proof for numerable bundles. Therefore, we state the result without further indication of proof.

THEOREM 2. Suppose $\left(p, p_{0}\right)$ is a numerable bundle pair over the cartesian product $B \times I$ of a space $B$ with the unit interval $I$. Let $r: B \times I \rightarrow B \times I$ be the map defined by $r(b, t)=(b, 0)$ for all $(b, t) \in$ $B \times I$. Then any pullback under $r$ of the bundle pair $\left(p, p_{0}\right)$ is isomorphic over $B \times I$ to $\left(p, p_{0}\right)$.

We will be concerned with pullbacks of the bundle pair $(q: Y \times Y \rightarrow$ $\left.Y, q_{0}: D(Y) \rightarrow Y\right)$ where $Y$ is a topological space, $\left.D(Y)=\{y, y) \mid y \in Y\right\}$ is the diagonal in $Y \times Y$, and $q: Y \times Y \rightarrow Y$ is the projection onto the first factor. The following proposition gives a necessary and sufficient condition for $\left(q, q_{0}\right)$ to be locally trivial (numerable). The condition may be thought of as a sort of local homogeneity condition.

Proposition 1. The bundle pair $\left(q, q_{0}\right)$ is locally trivial (numerable) iff there is a (numerable) open cover $\left\{U_{\alpha} \mid \alpha \in A\right\}$ of $Y$ and for each $\alpha \in A$ a continuous family $\left\{\phi_{\alpha x y} \mid(x, y) \in U_{\alpha} \times U_{\alpha}\right\}$ of homeomorphisms $\phi_{\alpha x y}: Y \rightarrow Y$ such that $\phi_{r x y}(x)=y$ for every $\alpha \in A$ and $(x, y) \in U_{\alpha} \times U_{\alpha}$.

(The family $\left\{\dot{\phi}_{\alpha x y} \mid(x, y) \in U_{\alpha} \times U_{\alpha}\right\}$ is continuous if $\dot{\phi}_{\alpha x y}(z)$ is a continuous function of the triple $(x, y, z)$.)

Proof. Suppose first that such a (numerable) covering and indexed family of homeomorphisms exists. For each $\alpha \in A$ choose an element $u_{\alpha} \in U_{\alpha}$ and let $F_{\alpha}=Y$ and $F_{0 \alpha}=\left\{u_{\alpha}\right\}$. Define $h_{\alpha}: q^{-1}\left(U_{\alpha}\right) \rightarrow U_{\alpha} \times F_{\alpha}$ by $h_{n}(x, y)=\left(x, \phi_{\alpha x u_{x}}(y)\right)$. Then $h_{\alpha}$ is a homeomorphism, $h_{\alpha}$ followed by projection onto $U_{\alpha}$ is simply $q \mid q^{-1}\left(U_{\alpha}\right)$, and $h_{\kappa}(x, y)=u_{\alpha}$ when $x=y$, so $h_{\alpha}\left(q_{0}^{-1}\left(U_{\alpha}\right)\right)=F_{0, \alpha}$. Thus $\left\{U_{\alpha} \mid \alpha \in A\right\}$ is a (numerable) trivializing cover for $\left(q, q_{0}\right)$. Conversely, suppose $\left\{U_{\alpha} \mid \alpha \in A\right\}$ a (numerable) open cover of $Y$ and $\left\{\left(h_{\alpha}: q^{-1}\left(U_{\alpha}\right) \rightarrow U_{\alpha} \times F_{\alpha}, h_{0 \alpha}: q_{0}^{-1}\left(U_{\alpha}\right) \rightarrow U_{\alpha} \times F_{0 \alpha}\right) \mid \alpha \in A\right\}$ a family of pairs of homeomorphisms such that $h_{0 \alpha}=h_{\alpha} \mid U_{\alpha} \times F_{0 \alpha}$ and $\pi_{\alpha} \circ h_{\alpha}(x, y)=q(x)$ for each $\alpha \in A$ and $(x, y) \in q^{-1}\left(U_{\alpha}\right)$, where $\pi_{\alpha}: U_{\alpha} \times F_{\alpha} \rightarrow$ $U_{\alpha}$ is the projection. For each $\alpha \in A$ and $(x, y) \in U_{\alpha} \times U_{\alpha}$ define $\phi_{\alpha x y}: Y \rightarrow Y$ by $\dot{\phi}_{\alpha x y}(z)=\pi_{\alpha}^{\prime} \circ h_{\alpha}^{-1}\left(y, \pi_{\alpha}^{\prime \prime} \circ h_{\alpha}(x, z)\right)$ where $\pi_{\alpha}^{\prime}: U_{\alpha} \times Y \rightarrow Y$ and $\pi_{\alpha}^{\prime \prime}: U_{\alpha} \times F_{\alpha} \rightarrow F_{\alpha}$ are the indicated projections. The map $\phi_{\alpha x y}$ is illustrated below. Then $\dot{\phi}_{x x y}$ is a homeomorphism, in fact $\dot{\phi}_{x x y}^{-1}=\dot{\phi}_{x y x}$, for each $\alpha \in A$ and $(x, y) \in U_{\alpha} \times U_{\alpha}$. Moreover $\dot{\phi}_{\alpha x y}(z)$ is continuous in $x, y, z$ simultaneously. Finally, suppose $z=x$. Then $(x, z) \in D(Y)$. This 


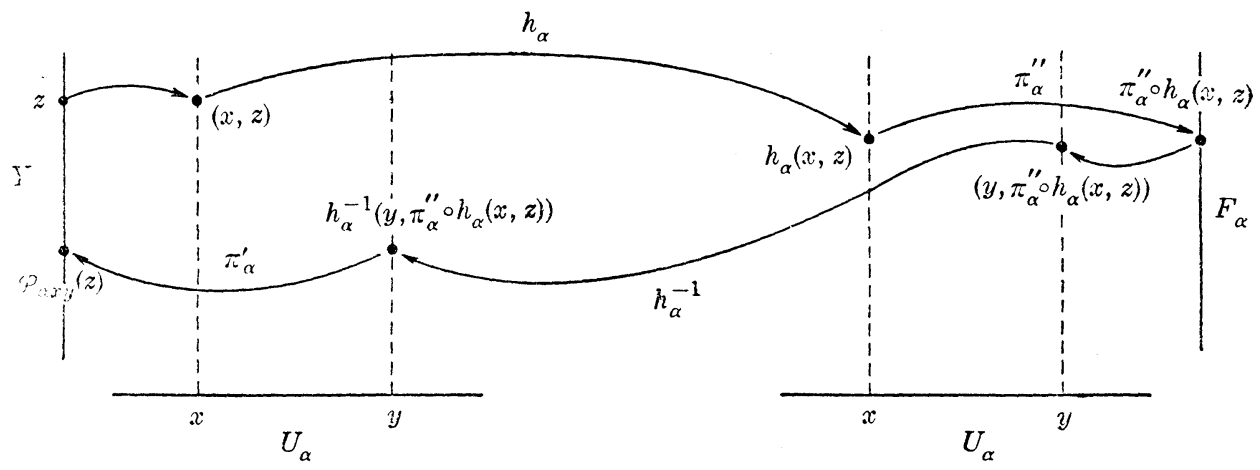

FIG. 1

implies that $h_{\alpha}(x, z) \in\{x\} \times F_{0}$, so $\pi_{\alpha}^{\prime \prime} \circ h_{\alpha}(x, z) \in F_{0}$, and therefore

$$
\left(y, \pi_{\alpha}^{\prime \prime} \circ h_{\alpha}(x, z)\right) \in\{y\} \times F_{0} .
$$

Hence $h_{\alpha}^{-1}\left(y, \pi_{\alpha}^{\prime \prime} \circ h_{\alpha}(x, z)\right) \in D(Y)$, and therefore $\pi_{\alpha}^{\prime}\left(h^{-1}\left(y, \pi_{\alpha}^{\prime \prime} \circ h_{a}(x, z)\right)=y\right.$. Thus $\phi_{\alpha x y}(x)=y$ for all $\alpha \in A$ and $(x, y) \in U_{\alpha} \times U_{\alpha}$.

Note that if $Y$ is a topological group then we may define $\phi_{x y}: Y \rightarrow$ $Y$ by $\dot{\phi}_{x y}(z)=y x^{-1} z$ for all $(x, y, z) \in Y \times Y \times Y$, so we obtain a trivializing cover for $\left(q, q_{0}\right)$ consisting of $Y$ alone. More important is the case where $Y$ is a manifold.

Proposition 2. If $Y$ is a topological manifold, then $(q: Y \times Y \rightarrow$ $\left.Y, q_{0}: D(Y) \rightarrow Y\right)$ is numerable.

Proof. Let $Y$ be a topological manifold. Then there is a family $\left\{\psi_{\alpha}: U_{\alpha} \rightarrow \mathbf{R}^{n} \mid \alpha \in A\right\}$ of charts for $Y$ such that $\left\{U_{\alpha} \mid \alpha \in A\right\}$ is numerable. For each $\alpha \in A$ and $(x, y) \in U_{\alpha} \times U_{\alpha}$ define $\dot{\phi}_{\alpha x y}: Y \rightarrow Y$ by

$$
\dot{\phi}_{a x y}(z)=\left\{\begin{array}{l}
z, \text { if } z \in Y-U_{\alpha} \\
\psi_{\alpha}^{-1}\left(\psi_{\alpha}(z)+\frac{\psi_{\alpha}(y)-\psi_{\alpha}(x)}{1+\left|\psi_{\alpha}(z)-\psi_{\alpha}(x)\right|}\right), \text { if } z \in U_{n}
\end{array}\right.
$$

and then apply the preceding proposition.

2. Proof of Theorem 1. Throughout this section we will assume that either the bundle pair $\left(q: Y \times Y \rightarrow Y, q_{0}: D(Y) \rightarrow Y\right)$ is numerable, or that it is locally trivial and $X$ is a paracompact space. A necessary and sufficient condition for the local triviality (numerability) of $\left(q, q_{0}\right)$ has been given in Proposition 1. In particular, $\left(q, q_{0}\right)$ will be numerable if $Y$ is a topological manifold.

LEMma. Suppose that $\left\{g_{t}: X \rightarrow Y \mid t \in I\right\}$ is a homotopy. Then there 
is an isotopy $\left\{h_{t}: X \times Y \rightarrow X \times Y \mid t \in I\right\}$ sush that

$$
h_{t}\left(x, g_{0}(x)\right)=\left(x, g_{t}(x)\right)
$$

for all $x \in X$ and $t \in I$.

Proof. Define $G: X \times I \rightarrow Y$ by $G(x, t)=g_{t}(x)$ for all $(x, t) \in X \times I$. Let $p: X \times I \times Y \rightarrow X \times I$ be the projection and $p_{0}$ : Graph $(G) \rightarrow X \times I$ its restriction to the graph of $G$, i.e., the set of all triples $(x, t, G(x, t))$ with $(x, t) \in X \times I$. Then it is easy to verify that $\left(p, p_{0}\right)$ is a pullback under $G$ of $\left(q, q_{0}\right)$. (To see this, define $G^{*}:(X \times I \times Y$, Graph $(G)) \rightarrow$ $(Y \times Y, D(Y))$ by $G^{*}(x, t, y)=(G(x, t), y)$. Therefore, under the assumptions at the beginning of this section, $\left(p, p_{0}\right)$ is numerable. Define $r: X \times I \rightarrow X \times I$ by $r(x, t)=(x, 0)$, and let $p_{0}^{\prime}$ be the restriction of $p$ to the space $\left\{(x, t, y) \in X \times I \times Y \mid y=g_{0}(x)\right\}$. Then $\left(p, p_{0}^{\prime}\right)$ is a pullback under $r$ of $\left(p, p_{0}\right)$. (Let $r^{*}(x, t, y)=(x, 0, y)$.) Hence, by Theorem $2,\left(p, p_{0}^{\prime}\right)$ is isomorphic to $\left(p, p_{0}\right)$. There is therefore a homeomorphism $H: X \times I \times Y \rightarrow X \times I \times Y$ such that $H\left(x, t, g_{0}(x)\right)=(x, t, G(x, t))$ for every $(x, t) \in X \times I$, and $p \circ H=p$. Thus, if we write $h_{t}(x, y)$ for the projection of $H(x, t, y)$ in $X \times Y$ we obtain the desired isotopy $\left\{h_{t}: X \times Y \rightarrow X \times Y \mid t \in I\right\}$.

We turn now to the proof of Theorem 1. We are given maps $f, g: X \rightarrow Y$ and maps $f^{\prime}, g^{\prime}: X \rightarrow Y$ homotopic to $f$ and $g$ respectively. We are also given a specific homotopy $\left\{g_{t} \mid t \in I\right\}$ from $g$ to $g^{\prime}$. We must find a homotopy $\left\{f_{t}: X \rightarrow Y \mid t \in I\right\}$ beginning at $f^{\prime}$ such that $\Gamma\left(f_{t}, g_{1-t}\right)=\Gamma\left(f^{\prime}, g^{\prime}\right)$ for all $t \in I$.

Let $\left\{h_{t}: X \times Y \rightarrow X \times Y \mid t \in I\right\}$ be an isotopy with the properties given in the lemma. Let $\pi: X \times Y \rightarrow Y$ be the projection. Then we define $f_{t}$ by

$$
f_{t}=\pi \circ h_{1-t} \circ h_{1}^{-1}\left(x, f^{\prime}(x)\right)
$$

for every $x \in X$ and $t \in I$. To see that $\left\{f_{t} \mid t \in I\right\}$ is the desired homotopy note first that $f_{0}(x)=\pi \circ h_{1} \circ h_{1}^{-1}\left(x, f^{\prime}(x)\right)=f^{\prime}(x)$ for every $x \in X$, so $\left\{f_{t} \mid t \in I\right\}$ begins at $f^{\prime}$. It remains to show that $\Gamma\left(f, g_{1-t}\right)=\Gamma\left(f_{0}, g_{1}\right)$, for all $t \in I$. Let $t \in I$, and suppose first that $x \in \Gamma\left(f_{t}, g_{1-t}\right)$, so $f_{t}(x)=$ $g_{1-t}(x)$. Then

$$
\begin{aligned}
f_{0}(x) & =\pi \circ h_{1} \circ h_{1-t}^{-1} \circ h_{1-t} \circ h_{1}^{-1}\left(x, f_{0}(x)\right) \\
& =\pi \circ h_{1} \circ h_{1-t}^{-1}\left(x, f_{t}(x)\right) \\
& =\pi \circ h_{1} \circ h_{1-t}^{-1}\left(x, g_{1-t}(x)\right) \\
& =\pi \circ h_{1}\left(x, g_{0}(x)\right) \\
& =\pi\left(x, g_{1}(x)\right) \\
& =g_{1}(x)
\end{aligned}
$$


so $x \in \Gamma\left(f_{0}, g_{1}\right)$. Conversely, suppose $x \in \Gamma\left(f_{0}, g_{1}\right)$ so $f_{0}(x)=g_{1}(x)$. Then

$$
\begin{aligned}
f_{t}(x) & =\pi \circ h_{1-t} \circ h_{1}^{-1}\left(x, f^{\prime}(x)\right) \\
& =\pi \circ h_{1-t} \circ h_{1}^{-1}\left(x, f_{0}(x)\right) \\
& =\pi \circ h_{1-t} \circ h_{1}^{-1}\left(x, g_{1}(x)\right) \\
& =\pi \circ h_{1-t}\left(x, g_{0}(x)\right) \\
& =\pi\left(x, g_{1-t}(x)\right) \\
& =g_{1-t}(x),
\end{aligned}
$$

so $x \in \Gamma\left(f_{t}, g_{1-t}\right)$.

3. Applications to coincidence theory. In this section we use some of the notation, definitions, and elementary results that are summarized in the second section of [3]. In particular, we will regard a homotopy $F$ of maps from $X$ into $Y$ as a function from the unit interval into $\operatorname{Map}(X, Y)$, with the proviso that $F(t)(x)$ be continuous in both its arguments. Throughout this section $Y$ will be a topological manifold (or, more generally, the bundle pair $\left(p: Y \times Y \rightarrow p_{0}: D(Y) \rightarrow\right.$ $Y$ ) will be assumed to be numerable.) We will let $f, g: X \rightarrow Y$ be maps. The class of all pairs $(F, G)$ of homotopies of maps from $X$ into $Y$ will be denoted, as in [3], by $A_{1}$; the subset of $\Delta_{1}$ consisting of those pairs for which $G$ is the constant homotopy at $g$ will be denoted by $\Delta_{1}^{*}$. Let $C$ be the minimum number of coincidence of $f^{\prime}$ and $g^{\prime}$ when $f^{\prime}$ and $g^{\prime}$ are allowed to vary over all maps homotopic to $f$ and $g$ respectively; let $C^{*}$ be the minimum number when just $f^{\prime}$ is allowed to vary but $g^{\prime}$ must remain fixed at $g$. Then Theorem 1 has the following immediate corollary.

\section{Corollary 1. $C=C^{*}$.}

We turn now to essentiality of coincidences and the Nielsen numbers.

Corollary 2. $x \in \Gamma(f, g)$ is $\Delta_{1}$ essential iff it is $\Delta_{1}^{*}$ essential.

Proof. If $f$ is $\Delta_{1}$ essential it is certainly $\Delta_{1}^{*}$ essential. Conversely, suppose $x \in \Gamma(f, g)$ is $\Delta_{1}^{*}$ essential, and let $F$ and $G$ be homotopies beginning at $f$ and $g$ respectively. According to Theorem 1 , there is a homotopy $F^{\prime}$ beginning at $F(1)$ such that for any $x \in X$ and any $t \in I$ we have $F^{\prime}(t)(x)=G(1-t)(x)$ iff $F(1)(x)=G(1)(x)$. Since $x$ is $\Delta_{1}^{*}$ essential it is $F F^{\prime}, g$ related to a point $x^{\prime} \in \Gamma\left(F^{\prime}(1), g\right)$. Since $F^{\prime}(1)(x)=$ $g(x)=G(0)(x)$, we have $F^{\prime}(1-t)\left(x^{\prime}\right)=G(t)\left(x^{\prime}\right)$ for all $t \in I$. Thus $x^{\prime} \in$ $\Gamma(F(1), G(1))$ and is $F^{\prime-1}, G$ related to itself. (The constant path at 
$x^{\prime}$ is a path for which $\left.\left.\left.\left[<F^{\prime-1}, x^{\prime}\right\rangle\right]=\left[<G, x^{\prime}\right\rangle\right]\right)$. It follows that $x$ is $\left(F F^{\prime \prime}\right) F^{\prime-1}, g G$ related to $x^{\prime}$. But $\left[\left(F F^{\prime}\right) F^{\prime-1}\right]=[F]$ and $[g G]=[G]$, so $x$ is $F, G$ related to $x^{\prime}$. It follows that $x$ is $\Delta_{1}$-essential.

Corollary 3. $N\left(f, g, \Delta_{1}\right)=N\left(f, g, \Delta_{1}^{*}\right)$, i.e., the $\Delta_{1}$ and $\Delta_{1}^{*}$ Nielsen numbers of $f$ and $g$ are equal.

Proof. By Corollary 2, each $\Delta_{1}^{*}$-essential coincidence class of $f$ and $g$ is also $A_{1}$-essential and conversely.

In the study of fixed points one is interested in finding a lower bound for the number of solutions to equations of the form $f(x)=x$, where $f: Y \rightarrow Y$ is allowed to vary throughout a homotopy class. In the study of roots one wants a lower bound for the number of solutions to equations of the form $f(x)=a$, where $f: X \rightarrow Y$ is again allowed to vary throughout a homotopy class, and $a$ is a given point in $Y$. In general coincidence theory, one wants a lower bound to the number of solutions to an equation of the form $f(x)=g(x)$, where $f: X \rightarrow Y$ is allowed to vary throughout one homotopy class and $g: X \rightarrow$ $Y$ throughout another. The first two equations are spəcial cases of the third (let $g=1_{Y}$ in the first and $g$ be the constant map in the second). But the first two theories are not, in general, spacial cases of the third-since in the third $g$ as well as $f$ is allowed to vary by a homotopy. Howevery our results show that when $Y$ is a manifold the first two theories are indeed special cases of the third. As an example we give an alternative proof of the following result announced in [2].

Proposition 3. Suppose $f: X \rightarrow Y$ a map of a topological space $X$ into a topological manifold $Y$, and suppose $a \in Y$. Then if the equation $f(x)=a$ has at least one $\Delta_{2}$-essential solution $\left(A_{2}\right.$ is the class of all pairs $(F, G)$ of homotopies of maps from $X$ into $Y$ in which $G$ is the constant homotopy at the constant map into $\alpha)$, then it has at least $R(f) \Delta_{2}$-essential solutions, where $R(f)$ is the order of the cokernel of the fundamental group homomorphism $f_{\sharp}: \pi(X) \rightarrow \pi(Y)$ induced by $f$.

Proof: Since $N\left(f, g, \Delta_{2}\right)>0$, there is an $x_{0} \in X$ with $f\left(x_{0}\right)=a$. Base the fundamental groups of $X$ and $Y$ at $x_{0}$ and $a$ respectively. Let $g: X \rightarrow Y$ be the constant map into $a \in Y$. Then $\Delta_{1}^{*}$ is $\Delta_{2}$, so Corollary 3 implies $N\left(f, g, \Delta_{1}\right)=N\left(f, g, \Delta_{2}\right)>0$. Thus, according to Theorem 1 of [3], $N\left(f, g, \Delta_{1}\right) \geqq J\left(f, g, \Delta_{1}\right)$, where, since $g_{\ddagger}: \pi\left(X, x_{0}\right) \rightarrow$ $\pi(Y, a)$ is trivial, $J\left(f, g, \Delta_{1}\right)$ is the number of elements of the cokernel of $f_{\sharp}$ that have representatives of the form [ $\left.\left\langle F, x_{0}\right\rangle\right]\left[\left\langle G, x_{0}\right\rangle\right]$, where $F$ is a homotopy from $f$ to itself and $G$ is a homotopy from $g$ to it- 
self. But since $g$ is the constant map, every loop $C$ in $Y$ at $a$ is of the form $\left[\left\langle G, x_{0}\right\rangle\right]$ where $G$ is a homotopy from $g$ to itself (set $G(t)(x)=C(t)$ for all $x \in X)$. Thus $J\left(f, g, \Delta_{1}\right)=$ order cokernel $f_{\sharp}=$ $R(f)$, so $N\left(f, g, \Delta_{2}\right) \geqq R(f)$. On the other hand [3, p. 557], we always have $R(f) \geqq N\left(f, g, \Delta_{2}\right)$, so $R(f)=N\left(f, g, \Delta_{2}\right)$.

We may also combine our results with one due to Schirmer [6] to provide a partial converse to Proposition 3.

Proposition 4. Suppose $X$ and $Y$ are triangulable manifolds of the same dimension $\geqq 3$. Then there is a map $f^{\prime \prime}$ homotopic to $f$ such that $f^{\prime \prime}(x)=a$ has exactly $N\left(f, g, \Delta_{2}\right)$ solutions, so, in particular, if $N\left(f, g, \Delta_{2}\right)>0$ then $f^{\prime \prime}(x)=a$ has exactly $R(f)$ solutions.

Proof. In [1, p. 109] a result of Schirmer's is translated into our terminology to give the following result: there is a map $f^{\prime}$ homotopic to $f$ and a map $g^{\prime}$ homotopic to the constant map $g$ such that $f^{\prime}(x)=$ $g^{\prime}(x)$ has exactly $N\left(f, g, \Delta_{1}\right)$ solutions. Theorem 1 and Corollary 3 therefore imply that there is a map $f^{\prime \prime}$ homotopic to $f^{\prime}$ and therefore to $f$ such that $f^{\prime \prime}(x)=a$ has exactly $N\left(f, g, \Delta_{2}\right)=N\left(f, g, \Delta_{1}\right)$ solutions.

\section{REFERENCES}

1. R. Brooks, Coincidences, roots, and fixed points, Doctoral Dissertation, University of California, Los Angeles, 1967.

2. - The number of roots of $f(x)=a$, Bull. Amer. Math. Soc., forthcoming.

3. - and R. F. Brown, A lower bound for the A-Nielsen number, Trans. Amer. Math. Soc., 143 (1969). 555, 564.

4. F. B. Fuller, The homotopy theory of coincidences, Doctoral Dissertation, Princeton University, 1951.

5. D. Husemoller, Fibre Bundles, McGraw-Hill, New York, 1966.

6. H. Schirmer, Mindestzahlen von Koinzidenzpunkten, J. Reine Angew. Math., 194 (1955), 21-39.

Received July 27, 1970.

BOWDOIN COLLEGE 


\section{PACIFIC JOURNAL OF MATHEMATICS}

\section{EDITORS}

H. SAMELSON

Stanford University

Stanford, California 94305

C. R. HOBBY

University of Washington

Seattle, Washington 98105
J. DugundJI

Department of Mathematics

University of Southern California

Los Angeles, California 90007

RICHARD ARENS

University of California

Los Angeles, California 90024

\section{ASSOCIATE EDITORS}

E. F. BeCKENBACH

B. H. NeumanN

F. WOLF

K. YosHIDA

\section{SUPPORTING INSTITUTIONS}

UNIVERSITY OF BRITISH COLUMBIA

CALIFORNIA INSTITUTE OF TECHNOLOGY

UNIVERSITY OF CALIFORNIA

MONTANA STATE UNIVERSITY

UNIVERSITY OF NEVADA

NEW MEXICO STATE UNIVERSITY

OREGON STATE UNIVERSITY

UNIVERSITY OF OREGON

OSARA UNIVERSITY
UNIVERSITY OF SOUTHERN CALIFORNIA STANFORD UNIVERSITY

UNIVERSITY OF TOKYO

UNIVERSITY OF UTAH

WASHINGTON STATE UNIVERSITY

UNIVERSITY OF WASHINGTON

AMERICAN MATHEMATICAL SOCIETY

NAVAL WEAPONS CENTER

Printed in Japan by International Academic Printing Co., Ltd., Tokyo, Japan 


\section{Pacific Journal of Mathematics}

\section{Vol. 40, No. $1 \quad$ September, 1972}

Alex Bacopoulos and Athanassios G. Kartsatos, On polynomials

approximating the solutions of nonlinear differential equations........

Monte Boisen and Max Dean Larsen, Prüfer and valuation rings with zero

divisors ..........................................

James J. Bowe, Neat homomorphisms

David W. Boyd and Hershy Kisilevsky, The Diophantine equation

$$
u(u+1)(u+2)(u+3)=v(v+1)(v+2) \ldots \ldots \ldots \ldots \ldots \ldots \ldots
$$

George Ulrich Brauer, Summability and Fourier analysis ...............

Robin B. S. Brooks, On removing coincidences of two maps when only one,

rather than both, of them may be deformed by a homotopy ............

Frank Castagna and Geert Caleb Ernst Prins, Every generalized Petersen

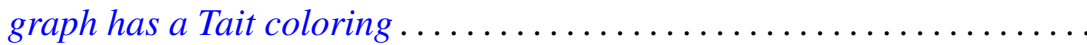

Micheal Neal Dyer, Rational homology and Whitehead products ..........

John Fuelberth and Mark Lawrence Teply, The singular submodule of a

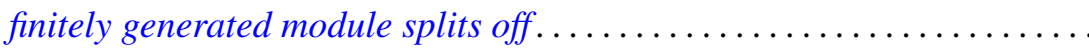

Robert Gold, $\Gamma$-extensions of imaginary quadratic fields ............ 83

Myron Goldberg and John W. Moon, Cycles in k-strong tournaments.......

Darald Joe Hartfiel and J. W. Spellmann, Diagonal similarity of irreducible

matrices to row stochastic matrices...............

Wayland M. Hubbart, Some results on blocks over local fields ..

Alan Loeb Kostinsky, Projective lattices and bounded homomorphisms....

Kenneth O. Leland, Maximum modulus theorems for algebras of operator

valued functions ...

Jerome Irving Malitz and William Nelson Reinhardt, Maximal models in the

language with quantifier "there exist uncountably many" ..

John Douglas Moore, Isometric immersions of space forms in space

forms.

Ronald C. Mullin and Ralph Gordon Stanton, A map-theoretic approach to

Davenport-Schinzel sequences ....................

Chull Park, On Fredholm transformations in Yeh-Wiener space. .

Stanley Poreda, Complex Chebyshev alterations ..............

Ray C. Shiflett, Extreme Markov operators and the orbits of Ryff. ...

Robert L. Snider, Lattices of radicals .....................

Ralph Richard Summerhill, Unknotting cones in the topological

category ................................

Charles Irvin Vinsonhaler, A note on two generalizations of $\mathrm{QF}-3 \ldots \ldots 229$

William Patterson Wardlaw, Defining relations for certain integrally

parameterized Chevalley groups...................

William Jennings Wickless, Abelian groups which admit only nilpotent

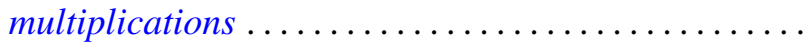

